\title{
Audit of venous thromboembolism prophylaxis administered to general surgical patients undergoing elective and emergency operations at National Hospital, Sri Lanka
}

\author{
*Migara Seneviratne ${ }^{1}$, Asanka Hemachandra ${ }^{2}$, Anuja Abayadeera ${ }^{3}$ \\ Specialist Registrar in Anaesthesia, University College London Hospital, United Kingdom ${ }^{l}$, \\ Registrar in Anaesthesiology, National Hospital Sri Lanka ${ }^{2}$, Senior Lecturer in Anaesthesia, \\ Faculty of Medicine, University of Colombo ${ }^{3}$
}

*Corresponding Author: dr_sene@yahoo.co.uk

Keywords: venous thromboembolism, prophylaxis, general surgery

\begin{abstract}
Venous thromboembolism (VTE) is a significant global cause for morbidity and mortality of surgical patients. Clinical guidelines written by the National Institute of Clinical Excellence (NICE) in Great Britain is available to manage this problem. The Sri Lanka Health Service currently has no such guidelines.
\end{abstract}

\begin{abstract}
We conducted a retrospective audit of current practice of VTE prophylaxis in general surgical patients having operations at the National Hospital of Sri Lanka (NHSL). Ethical approval was obtained. Clinical records were perused to audit risk assessment and management by clinical teams involved. A data form addressing NICE guidelines was used and statistically analysed.
\end{abstract}

Of 244 cases listed in the theatre register 154 case notes were located. We identified that clinical staff were deficient in obtaining information required to classify patient risk of VTE. Only 13\% of cases had a Body Mass Index calculated. Sub optimal records were evident in the surgical, anaesthetic and nursing notes. Majority of cases had one or more risk factors for VTE. Most patients underwent elective surgery under general anaesthesia. $71.4 \%$ of patients had an indication for mechanical, pharmacological or both methods for VTE prophylaxis. None of the patients requiring only mechanical or only pharmacological prophylaxis received any. Only 5.9\% of patients were correctly administered both forms of prophylaxis when required. Administration of mechanical prophylaxis was not documented in a single case.

We suggest changes at an institutional/ individual level. These should aim to improve proper note binding, clinical record keeping, availability of guidelines/ protocols and a multi-disciplinary approach to risk assessment. Hospitals should ensure a minimum standard availability of mechanical prophylaxis. Anaesthetists should cover VTE risk during pre-operative assessment. Mechanical prophylaxis commenced in theatre should be documented in the anaesthetic chart. Post-operative visit should include a risk assessment for ongoing risk of VTE. Measures should be taken to re-audit clinical practice according to set guidelines in future.

VTE encompasses both deep vein thrombosis and pulmonary embolism which lead to significant patient morbidity and mortality. Surgery is associated with a substantial risk of VTE within the first post operative week in comparison to the background incidence of VTE in the general population ${ }^{1}$. This risk peaks at the third post operative week and remains higher than the un-operated patient for 12 months. Degree of VTE risk for individual patients is dependent on multiple factors. Clinical guidelines and protocols have been written by the NICE to identify this risk and administer appropriate prophylaxis ${ }^{2}$. A.H Sheriffdeen highlighted the importance for VTE prophylaxis in Sri Lanka in 1984 and further re-iterated it in $2001^{3,4}$. 10 years later the National Health Service in Sri Lanka is still short of (freely accessible) guidelines on VTE prophylaxis. This audit is a comparison of clinical risk assessment and provision for VTE prophylaxis at NHSL against NICE guideline standards. 


\section{Method}

\section{Audit framework}

This included patient selection, time frame, data collection and statistical analysis. Ethics approval and permission was obtained from the NHSL Ethics Committee and consultant surgeons. A patient group having general surgical operations in theatres A, B and D at the NHSL from $1^{\text {st }}-31^{\text {st }}$ July 2010 was chosen. Cases identified from the theatre registers were submitted to clinical records for location. Data entry was done consistently by two authors using a data collection sheet designed to address NICE guidelines. Sections covering surgical and trauma patients of the NICE guidelines were used with regards to risk assessments for both VTE and bleeding. VTE prophylaxis recommendations were taken from the categories Gastrointestinal, Day Surgery and Other Surgery of the NICE Guidelines. The completed data sheets were statistically analysed using SPSS 15 software and a unique spreadsheet.

\section{Assessment of VTE risk and requirement for prophylaxis}

The NICE guidelines state that the following clinical factors increase the risk of VTE;

- total anaesthetic + surgical time $>90$ minutes

- surgery involving pelvis or lower limb and total anaesthetic + surgical time $>60$ minutes

- Acute surgical admission with inflammatory or intra-abdominal condition

- Expected to have significant reduction in mobility ( $\geq 3$ days)

- $\geq 1$ individual VTE risk factors present

For uniformity the authors categorised patients into the following risk groups of VTE; Low Risk $(<1$ Clinical Factor), Moderate Risk $(=1$ Clinical Factor) and High Risk $(\geq 2$ Clinical Factors).

Individual VTE risk factors listed in the NICE guidelines were:

- Malignancy

- Age $>60$

- Critical care admission

- Dehydration

- Thrombophilias

- Obesity $\left(\mathrm{BMI}>30 \mathrm{~kg} / \mathrm{m}_{2}\right)$
- $\quad \geq 1$ significant medical co-morbidities

- Personal history or $1^{\text {st }}$ degree relative with history of VTE

- Use of HRT or oestrogen containing contraceptive therapy

- Varicose veins with phlebitis

Clinical judgement from information available in the notes was used for the assessment of dehydration. Significant medical co-morbidities were based on factors such as end organ damage, multiple medications, long duration of condition or if the condition directly led to their admission. The response to personal history or $1^{\text {st }}$ degree relative with history of VTE was based on whether any attempt was made to find out about family history. The admission notes and drug chart were both used to identify any use of HRT or oestrogen containing contraceptive therapy.

\section{VTE prophylaxis}

The authors decided on the following treatment options for the VTE risk groups; Early mobilisation for Low Risk, mechanical VTE prophylaxis for Moderate Risk and both mechanical and pharmacological prophylaxis for High Risk. When the mechanical method was contraindicated or impractical a pharmacological method was recommended. When the risk of bleeding was high the pharmacological method should be avoided.

\section{In-patient management}

Period of immobility and fluid administration was obtained where possible from clinical documentation. Physiotherapy involvement was documented as obtained if the medical or nursing notes stated that any form of physiotherapy had been done or requested.

\section{Results}

\section{Clinical records}

154 of the 244 cases identified from theatre registers were tracked by clinical records and analysed. The remaining 90 cases were either not located or incomplete and excluded from the audit.

\section{Study population demographics}

The name, age and sex were documented in all the study participants. $116 \quad(75.3 \%)$ were females. $27(17.5 \%)$ were above 60 years of age. Weight was documented in $31(20.1 \%)$ patients and height in $19(12.3 \%)$ patients. BMI could be 
calculated in $19(12.3 \%)$ patients. From these 19 patients the BMI showed 2 patients to be clinically obese.

\section{Nature of admissions and duration of hospital} stay

122 cases $(79.2 \%)$ were admitted for elective procedures. The remaining 32 (20.8\%) were emergency operations. The duration of stay in hospital was identified in 145 (94.2\%) cases. The mean duration for this group was 7.68 days and the median duration 5 days (range 1- 81 days). The remaining $9(5.8 \%)$ had no documented date of discharge.

Table 1: Distribution of the individual VTE risk factors of the sample

\begin{tabular}{|c|c|c|c|c|c|c|}
\hline \multirow[t]{2}{*}{ Risk feature } & \multicolumn{2}{|l|}{ No } & \multicolumn{2}{|l|}{ Yes } & \multicolumn{2}{|l|}{ Total } \\
\hline & $\begin{array}{l}\text { Num } \\
\text { ber }\end{array}$ & $\%$ & $\begin{array}{l}\text { Numb } \\
\text { er }\end{array}$ & $\%$ & $\begin{array}{l}\text { Numbe } \\
r\end{array}$ & $\%$ \\
\hline Cancer & 134 & 87.0 & 20 & 13.0 & 154 & 100.0 \\
\hline Age $>60$ years & 127 & 82.5 & 27 & 17.5 & 154 & 100.0 \\
\hline $\begin{array}{l}\text { Critical care } \\
\text { admission }\end{array}$ & 146 & 94.8 & 8 & 5.2 & 154 & 100.0 \\
\hline Dehydration & 129 & 83.8 & 25 & 16.2 & 154 & 100.0 \\
\hline Thrombophilias & 154 & $\begin{array}{l}100 . \\
0\end{array}$ & 0 & 0.0 & 154 & 100.0 \\
\hline $\begin{array}{l}\text { Obesity } \\
\left(\text { BMl }>30 \mathrm{~kg} / \mathrm{m}^{2}\right)\end{array}$ & 17 & 11.0 & 2 & 1.3 & 19 & 12.3 \\
\hline Co morbidities & 92 & 59.7 & 62 & 40.3 & 154 & 100.0 \\
\hline History of VTE & 49 & 31.8 & 1 & 0.6 & 50 & 32.5 \\
\hline HRT / OCP* & 110 & 98.2 & 2 & 1.8 & 112 & 96.5 \\
\hline Varicose veins & 143 & 92.9 & 11 & 7.1 & 154 & 100.0 \\
\hline
\end{tabular}

* HRT / OCP - this was calculated for those at risk, being the females in this group

Due to poor documentary evidence it was not possible to identify accurately the number of patients who had $\geq 1$ individual VTE risk factor. The poorest documented evidence was obtained for obesity (BMI $>30 \mathrm{~kg} / \mathrm{m}^{2}$ ) which was only available for $19(12.3 \%)$ of the total population. The second lowest evidence was obtained in relation to history of VTE which was only available on $50(32.5 \%)$ of the population. Documentary evidence for 4 women regarding HRT/ OCP was also not available.
Table 2: Risk profile for bleeding

\begin{tabular}{|c|c|c|c|c|}
\hline \multirow[t]{2}{*}{ Risk Feature } & \multicolumn{2}{|l|}{ No } & \multicolumn{2}{|l|}{ Yes } \\
\hline & Number & $\%$ & Number & $\%$ \\
\hline Active bleeding & 151 & 98.1 & 3 & 1.9 \\
\hline $\begin{array}{l}\text { Acquired bleeding } \\
\text { disorder }\end{array}$ & 152 & 98.7 & 2 & 1.3 \\
\hline $\begin{array}{l}\text { Anticoagulant use / INR } \\
>2\end{array}$ & 153 & 99.4 & 1 & 0.6 \\
\hline LP / Epidural / Spinal & 150 & 97.4 & 4 & 2.6 \\
\hline Stroke & 154 & 100 & 0 & 0.0 \\
\hline Thrombocytopenia & 152 & 98.7 & 2 & 1.3 \\
\hline Systolic hypertension & 152 & 98.7 & 2 & 1.3 \\
\hline $\begin{array}{l}\text { Inherited bleeding } \\
\text { disorders }\end{array}$ & 154 & 100 & 0 & 0.0 \\
\hline
\end{tabular}

Of the total 154 cases only 14 cases (9\%) had any risk of bleeding.

\section{Nature and duration of surgery (anaesthetic and surgical time)}

The sample population included a wide range of surgical procedures. The most reported surgery was thyroidectomy, with laparoscopic cholecystectomy the second highest. The duration was only recorded for $107(69.5 \%)$ of the patients. Out of these the mean was 101 minutes and median of 90 minutes (Range 20 360 minutes). 43 cases had a duration $>90$ minutes. Of the 7 lower limb operations 2 cases had a duration $>60$ minutes.

\section{Type of anaesthesia}

Of the total 154 persons who underwent surgery, 5 patients (3.2\%), had no recorded mode of anaesthesia. For the remainder, General anaesthesia (GA) was mostly used $(n=124$, $80.5 \%$ ). Regional anaesthesia (RA) was used in 20 cases $(13.0 \%)$. Both GA and RA were used in 4 cases $(2.6 \%)$. Local anaesthesia was used in 1 case.

\section{Recommended and administered VTE prophylaxis}

The numbers obtained for VTE risk were: Low Risk 44 patients (28.6\%), Moderate Risk 66 patients (42.9\%) and High Risk 44 patients $(22.1 \%)$ 
Table 3: Administered VTE prophylaxis in relation to recommended prophylaxis

\begin{tabular}{|c|c|c|c|c|c|}
\hline \multirow[t]{2}{*}{ VTE Prophylaxis } & \multirow[t]{2}{*}{ Total } & \multicolumn{2}{|c|}{$\begin{array}{l}\text { Mechanical } \\
\text { administered }\end{array}$} & \multicolumn{2}{|c|}{$\begin{array}{l}\text { Pharmacological } \\
\text { administered }\end{array}$} \\
\hline & & Number & $\%$ & Number & $\%$ \\
\hline Not indicated & 44 & 0 & 0.0 & 0 & 0.0 \\
\hline $\begin{array}{l}\text { Only Mechanical } \\
\text { (M) }\end{array}$ & 66 & 0 & 0.0 & 1 & 1.5 \\
\hline $\begin{array}{l}\text { Only } \\
\text { Pharmacological }(P)\end{array}$ & 10 & 0 & 0.0 & 0 & 0.0 \\
\hline Both $\mathrm{M}$ and $\mathrm{P}$ & 34 & 2 & 5.9 & 5 & 14.7 \\
\hline Total & 154 & 2 & 1.3 & 6 & 3.9 \\
\hline
\end{tabular}

110 patients $(71.4 \%)$ were identified as needing VTE prophylaxis. None of the 66 patients requiring only mechanical prophylaxis had documented evidence of receiving any. Likewise, none of the 10 patients needing only pharmacological prophylaxis received any. From the 34 patients identified as needing both types only 2 (5.9\%) had both forms of prophylaxis administered.

Table 4: Pharmacological VTE prophylaxis administered to the 6 patients

\begin{tabular}{|lccccc|}
\hline Agent & $\begin{array}{l}\text { Pre/ } \\
\text { Post }\end{array}$ & Modality & Duration & Dose & Frequency \\
Enoxaparin & Post & SC & 16 days & $20 \mathrm{mg}$ & Once daily \\
Heparin & Post & SC & 4 days & 5000 & Twice daily \\
& & & & & \\
Enoxaparin & Post & SC & NA days & $20 \mathrm{mg}$ & Once daily \\
& & & & $40 \mathrm{mg}$ & Once daily \\
Enoxaparin & Post & SC & 1 day & $20 \mathrm{mg}$ & Once daily \\
& & & & & \\
Tinzaparine & Post & SC & 7 days & 3500 & Once daily \\
& & & & & \\
& & & & &
\end{tabular}

\section{Inpatient management and discharge}

Duration of immobility was identified in 129 $(83.8 \%)$ of the patients. The range was 6 hours to 30 days (Mean 2.43 days/ median 1 day). 28 cases $(21.7 \%)$ had a period of immobility $\geq$ 3days. 126 cases had documented evidence of receiving fluids for the prevention of dehydration.
Table 5: Administration of physiotherapy in relation to the duration of immobility

\begin{tabular}{|lrrrrr|}
\hline $\begin{array}{l}\text { Duration of } \\
\text { immobility }\end{array}$ & \multicolumn{2}{l}{ No Physiotherapy } & $\begin{array}{l}\text { Received } \\
\text { Physiotherapy }\end{array}$ & Total \\
& Number & $\%$ & Number & $\%$ & \\
$<3$ days & 83 & 82.2 & 18 & 17.8 & 101 \\
$\geq 3$ days & 12 & 42.9 & 16 & 57.1 & 28 \\
Total & 95 & 73.6 & 34 & 26.4 & 129 \\
\hline
\end{tabular}

34 cases in total received physiotherapy. 12 cases (42.9\%) with $\geq 3$ days immobility did not receive physiotherapy. None of the 154 cases had documentary evidence that advice on VTE signs and symptoms were given to patients prior to discharge.

\section{Discussion}

Case notes tracking was limited reducing study number. This could have implications on the power of statistical analysis. The notes analysed showed a broad range of variables covered within the study population.

Only 19 patients had enough data for BMI calculation indicating a very poor level of attention given to measurement of weight and height. These variables should be measured irrespective of a patient's disease status.

9 cases (5.8\%) had no documented date of discharge. 47 cases $(30.5 \%)$ had no recorded duration of surgery. 5 patients $(3.2 \%)$ had no documented mode of anaesthesia in the notes and the period of immobility could not be identified in 25 cases $(16.2 \%)$. These results strongly indicate that clinical record keeping is sub optimal.

The audit group had risk factors for VTE. 43 cases had a total anaesthetic and surgical time > 90 minutes. 2 lower limb surgical procedures had an anaesthetic and surgical time $>60$ minutes. 32 cases $(20.8 \%)$ had emergency operations. 28 cases had a period of immobility $\geq 3$ days. In the category, Individual VTE risk factors, the low rate of data available made it impossible to identify accurately the total number of patients who had $\geq 1$ individual VTE risk factor. It is possible that significant negative findings from the initial medical clerking are not being documented by medical and nursing staff. This shows limited emphasis on the importance of VTE risk assessment. 
Only 14 cases $(9 \%)$ had any risk of bleeding. This proves that bleeding as a relative risk or contraindication to pharmacological VTE prophylaxis affected only a minority of cases.

The mechanical VTE prophylaxis methods recommended by NICE include; anti-embolism stockings, foot impulse devices and intermittent pneumatic compression devices. These are not freely available in the NHSL. Patients are expected to bring their own anti-embolism stockings. It should still be expected that when available they are used appropriately and documented in the nursing and medical notes. The number of patients that did receive pharmacological VTE was too small for any further statistical analysis.

Since completion of this audit in July 2010 the authors would like to point out that NICE revised the guidelines on VTE prophylaxis in May 2011 to clarify the use of extended pharmacological prophylaxis in specific patient groups.

\section{Conclusion}

The audit showed significant deficiencies in record keeping and clinical assessment of VTE risk in the population group. The study population had moderate $(42.9 \%)$ and high (22.1\%) risk of VTE. $98 \%$ of patients did not receive appropriate VTE prophylaxis.

\section{Recommendations}

The NHSL should aim to improve the process of proper note binding, tracking and clinical record keeping. Guidelines and protocols for VTE prophylaxis should be created and freely available. A multi-disciplinary approach to VTE risk assessment should be encouraged. Mandatory clinical staff training and awareness should be initiated. As a minimum standard, mechanical prophylaxis should be available for all patients. Prophylaxis should be commenced preoperatively and reassessed on a daily basis.
The clerking medical officer should document individual VTE risk factors as stated by the guidelines. Immobility and dehydration should be documented and treated. On discharge all at risk patients should be provided with information on signs and symptoms of VTE. A leaflet on VTE would be helpful in this process.

Recommendations for the anaesthetist include: VTE risk assessment during pre-operative consultation, documentation of any prophylaxis commenced in theatre and continued VTE risk assessment during subsequent post operative visits.

\section{References}

1. Sweetland S, Green J, Liu B et al; Duration and magnitude of the postoperative risk of venous thromboembolism in middle aged women: Prospective cohort study. British Medical Journal, 2009 Dec 3; 339:b4583.

2. CG92 Venous Thromboembolism - Reducing the Risk: NICE guideline;

http://guidance.nice.org.uk/CG92/NICEGuidanc e/pdf/English.

3. Sheriffdeen AH; Vascular Emergencies. Sri Lankan Family Physician 1981; 8: 131-49.

4. Sheriffdeen AH, Wijeyaratne M, Deep vein thrombosis in Sri Lankans- time to take notice. Ceylon Medical Journal. 2001 Mar; 46(1):3-5. 